\title{
Wirtinger presentations for higher dimensional manifold knots obtained from diagrams
}

\author{
by \\ Seiichi Kamada (Osaka and Mobile, AL)
}

\begin{abstract}
A Wirtinger presentation of a knot group is obtained from a diagram of the knot. T. Yajima showed that for a 2-knot or a closed oriented surface embedded in the Euclidean 4-space, a Wirtinger presentation of the knot group is obtained from a diagram in an analogous way. J. S. Carter and M. Saito generalized the method to non-orientable surfaces in 4 -space by cutting non-orientable sheets of their diagrams by some arcs. We give a modification to their method so that one does not need to find and describe such arcs on the diagram. This method is easily generalized to higher dimensional manifold knots, which may not be locally flat.
\end{abstract}

1. Introduction. A Wirtinger presentation of a knot group is obtained from a diagram of the knot (cf. $[2,4,20,21])$. For a 2 -knot or a closed oriented surface $M$ in $\mathbb{R}^{4}$, a Wirtinger presentation of the knot group $\pi_{1}\left(\mathbb{R}^{4}-M\right)$ is obtained from a (broken surface) diagram in an analogous way. Consider a diagram of $M$ in $\mathbb{R}^{3}$, and label the sheets of the diagram by $x_{1}, \ldots, x_{s}$, where $s$ is the number of sheets. Each $x_{i}$ is regarded as a meridian element of the knot group $\pi_{1}\left(\mathbb{R}^{4}-M\right)$ and the knot group is generated by $x_{1}, \ldots, x_{s}$. Each double curve (connected component of the double point set in $\mathbb{R}^{3}$ ) induces a relator of the form $x_{i} x_{j} x_{i}^{-1} x_{l}^{-1}$ or $x_{i} x_{j}^{-1} x_{i}^{-1} x_{l}$, where $x_{i}$ is the label of the upper sheet and $x_{j}, x_{l}$ are the lower sheets around the double curve. The exponents are the signs of the intersections of the oriented sheets $x_{i}, x_{j}, x_{l}$ and a small loop around the double curve (cf. [3, 25]). For a non-orientable surface in $\mathbb{R}^{4}$, this method does not apply directly. If a sheet of a broken surface diagram contains an orientation-reversing loop, then one cannot assign a meridian element to the sheet. This happens even

2000 Mathematics Subject Classification: Primary 57Q45; Secondary 57M05, 57M25.

Key words and phrases: Wirtinger presentation, knot group, higher dimensional knot, knot diagram, broken surface diagram.

The author is supported by a Fellowship from the Japan Society for the Promotion of Science. 
in diagrams with simple configurations as in Figure 1 (the diagrams present a standard projective plane and a standard Klein bottle in $\mathbb{R}^{4}$ ). The first diagram has a single sheet which is a Möbius band. The second has two sheets; one is a disk and the other is a punctured Klein bottle. To avoid such a bad situation, J. S. Carter and M. Saito divided the non-orientable sheets into orientable pieces by some arcs satisfying a certain condition (see [3]). One has to find such arcs and describe them on the diagram. This process is a little bothersome when the configuration of the diagram is not simple. In this paper we give an alternative method to divide the non-orientable sheets, which is quite elementary. In fact, the diagram itself gives the information on the division, and the argument is valid in higher dimensional cases. Moreover our argument does not require a surface in $\mathbb{R}^{4}$, or higher dimensional manifold knot, to be locally flat. We work in the PL (or smooth) category.
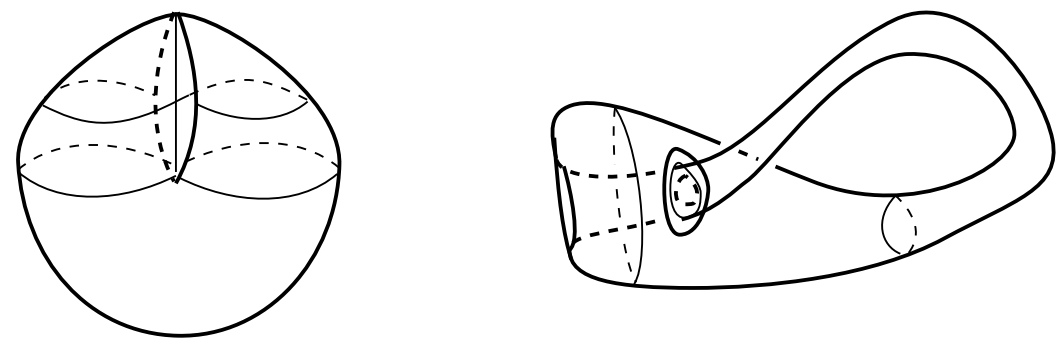

Fig. 1

A method to obtain a Wirtinger presentation for PL and locally flatly (or smoothly) embedded surfaces in $\mathbb{R}^{4}$ using diagrams was given by T. Yajima [25] (based on an idea of [16]) and a clear exposition (without a proof) is found in [3] including the non-orientable case. Existence of a Wirtinger presentation for such surfaces in $\mathbb{R}^{4}$ had been known before using diagrams. For example, Fox's method in [5] gives such a presentation. A similar method was used in [17]. The latter method works quite well if a surface in $\mathbb{R}^{4}$ is given in the motion picture form and the configuration of the surface satisfies a certain condition as on p. 134 of $[5]$. It is proved in $[9,12]$ that any (PL locally flat or smooth) surface in $\mathbb{R}^{4}$ is deformable into such a form. Existence of a Wirtinger presentation for higher dimensional orientable smooth manifold knots was stated by J. Simon in [23]. His idea is to use Fox's method inductively (the details for the 2-knot case are in [24], for example). Yajima [27] proved algebraically that the Kervaire conditions [14, 15] imply existence of a Wirtinger presentation, and hence a higher dimensional orientable (smooth or PL locally flat) manifold knot group has a Wirtinger presentation if the group has a trivial second homology (there exist a lot of manifold knot groups with non-trivial second homology [1, 7, 18, 19, 23]). 
For related topics on higher dimensional manifold knot groups, we refer to $[6,7,8,10,11,14,15,23,26,27]$.

The author thanks J. S. Carter, J. H. Przytycki, A. Kawauchi and D. Silver for valuable and fruitful conversations and comments. He also thanks the Department of Mathematics and Statistics, University of South Alabama for the hospitality during his visit.

2. Regular sheets. Let $M$ be a closed (not necessarily connected) $n$-manifold embedded piecewise linearly (or smoothly) in $\mathbb{R}^{n+2}$, and let $p: \mathbb{R}^{n+2}=\mathbb{R}^{n+1} \times \mathbb{R} \rightarrow \mathbb{R}^{n+1}$ be the projection. We suppose that $M$ is in general position with respect to the projection $p$. The singularity set $\Delta$ that is the closure of the multiple point set $\left\{y \in \mathbb{R}^{n+1}|| p^{-1}(y) \cap M \mid>1\right\}$ in $\mathbb{R}^{n+1}$ (or its preimage in $M$ ) is naturally regarded as an $(n-1)$-dimensional stratified complex. It is well known that the $(n-1)$-dimensional strata consist of transverse double points; we call them the double point strata and denote by $\Delta^{1}$. (This is seen by a general position argument which does not require $M$ to be locally flat, cf. [22].) Lower dimensional strata are in general complicated. For $n=2$, the 0 -dimensional strata consist of triple points and branch points if $M$ is locally flat (cf. [3, 13]); if $M$ is not locally flat, then there may be some cone points over classical knot diagrams in small 2 -spheres in $\mathbb{R}^{3}$. For our purpose, classification of lower dimensional strata is not required at all since they do not contribute to the knot group.

The singularity set $\Delta$ divides $p(M)$ (or $M$ in the preimage) into some pieces. Each piece (connected component of $p(M)-\Delta$ ) is an open $n$-manifold embedded in $\mathbb{R}^{n+1}$ consisting of regular points of $p(M)$, which we call an open regular sheet. Let $N(\Delta)$ be a regular neighborhood of $\Delta$ in $\mathbb{R}^{n+1}$. We call a component of $\operatorname{cl}(p(M)-N(\Delta))$ a regular sheet, where cl means closure. A regular sheet is a deformation retract of an open regular sheet. For example, the first (broken surface) diagram in Figure 1 has a single regular sheet which is a 2-disk. The second has two sheets; one is a 2-disk and the other is a punctured annulus.

\section{LEMma 1. (Open) regular sheets are 2-sided (or co-orientable).}

Proof. If $M$ is orientable, each (open) regular sheet is orientable and hence it is co-orientable in $\mathbb{R}^{n+1}$. If $M$ is non-orientable and if there is a non-orientable regular sheet $\Sigma$, take an orientation-reversing loop, say $c$, in the interior of the sheet $\Sigma$. We may assume that it is a simple loop (if $\operatorname{dim}(M)>3$, it is obvious; if $\operatorname{dim}(M)=2$, modify the loop if necessary). Push the loop $c$ out of $\Sigma$ in $\mathbb{R}^{n+1}$ along the normal direction, obtaining a loop $c^{\prime}$. Since $c$ is an orientation-reversing loop of $\Sigma$, the regular neighborhood $N\left(c ; \mathbb{R}^{n+1}\right)$ of $c$ in $\mathbb{R}^{n+1}$ is a twisted $I$-bundle over $N(c ; \Sigma)$ and hence we may assume that $\left|c^{\prime} \cap N(c ; \Sigma)\right|=1$. On the other hand, $\left|c^{\prime} \cap p(M)\right|=0$ 
$\bmod 2$, since any $\mathbb{Z}_{2}$-intersection number of cycles vanishes in $\mathbb{R}^{n+1}$. Hence $c^{\prime}$ has another intersection with $p(M)$ off $N(c ; \Sigma)$. This contradicts the fact that $c$ is a loop on $\Sigma$.

3. How to get a presentation. Let $M, p$ and $\Delta$ be as before. By a diagram of $M$ we mean the image $p(M)$ equipped with over-under information at each transverse double point. The knot group $\pi_{1}\left(\mathbb{R}^{n+2}-M\right)$ has the following "Wirtinger type" presentation: Let $\Sigma_{1}, \ldots, \Sigma_{s}$ be the regular sheets of the diagram of $M$, where $s$ is the number of regular sheets. By Lemma 1 , they are co-orientable in $\mathbb{R}^{n+1}$. Fix a co-orientation of each regular sheet. Generators of the group presentation are $x_{1}, \ldots, x_{s}$, which are represented by meridian loops of the regular sheets with a base point $*$ in $\mathbb{R}^{n+2}$ (explained later). Relators of the group presentation correspond to the double point strata and are of the form $x_{i} x_{j}^{\varepsilon_{1}} x_{k}^{\varepsilon_{2}} x_{l}^{\varepsilon_{3}}$ and $x_{i} x_{k}^{\varepsilon_{2}}$ where $\varepsilon_{1}, \varepsilon_{2}, \varepsilon_{3} \in\{1,-1\}$. Precisely speaking, for each double point stratum, consider a small loop in $\mathbb{R}^{n+1}$ intersecting the four regular sheets around the stratum, say $\Sigma_{i}, \Sigma_{j}, \Sigma_{k}, \Sigma_{l}$. By changing the starting point and the orientation of the loop if necessary, we may assume that $\Sigma_{i}, \Sigma_{j}, \Sigma_{k}, \Sigma_{l}$ appear in this order along the loop, $\Sigma_{i}$ and $\Sigma_{k}$ are above $\Sigma_{j}$ and $\Sigma_{l}$, and the loop intersects $\Sigma_{i}$ in the direction of the co-orientation of $\Sigma_{i}$. Then we have two relators $x_{i} x_{k}^{\varepsilon_{2}}$ and $x_{i} x_{j}^{\varepsilon_{1}} x_{k}^{\varepsilon_{2}} x_{l}^{\varepsilon_{3}}$ (or equivalently $x_{i} x_{k}^{\varepsilon_{2}}$ and $x_{i} x_{j}^{\varepsilon_{1}} x_{i}^{-1} x_{l}^{\varepsilon_{3}}$ ), where $\varepsilon_{1}, \varepsilon_{2}, \varepsilon_{3}$ are +1 (or -1 , resp.) if the loop intersects $\Sigma_{j}, \Sigma_{k}, \Sigma_{l}$ in the direction of (or in the opposite direction to, resp.) their respective coorientations.

THEOREM 2. The group presentation described above is a presentation of the knot group $\pi_{1}\left(\mathbb{R}^{n+2}-M\right)$. In particular, any $P L$ (or smooth) manifold knot group has a Wirtinger type presentation.

REMARK. Suppose that there is a relator $x_{i} x_{j}^{\varepsilon_{1}} x_{i}^{-1} x_{l}^{\varepsilon_{3}}$ with $\varepsilon_{3}=\varepsilon_{1}$ in the above presentation. If one prefers that $\varepsilon_{3}=-\varepsilon_{1}$, then one has to introduce a new generator, say $x_{l}^{\prime}$, and a relator $x_{l}^{\prime} x_{l}$ to the presentation, so that the relator $x_{i} x_{j}^{\varepsilon_{1}} x_{i}^{-1} x_{l}^{\varepsilon_{3}}$ may be replaced with $x_{i} x_{j}^{\varepsilon_{1}} x_{i}^{-1} x_{l}^{\prime-\varepsilon_{1}}$.

For a given Wirtinger type presentation, it is easy to construct a PL locally flat (or smooth) surface in $\mathbb{R}^{4}$ whose knot group has that presentation (see 14.2.1 of [11]). Thus we have the following.

Corollary 3. For any $P L$ (or smooth) manifold knot $M$ in $\mathbb{R}^{n+2}$, there exists a PL locally flat (or smooth) surface $F$ in $\mathbb{R}^{4}$ such that $\pi_{1}\left(\mathbb{R}^{n+2}-M\right)$ $\cong \pi_{1}\left(\mathbb{R}^{4}-F\right)$.

4. Proof of Theorem 2. First we explain the elements $x_{1}, \ldots, x_{s}$ of $\pi_{1}\left(\mathbb{R}^{n+2}-M\right)$. Let $\Sigma=\operatorname{cl}(p(M)-N(\Delta))$, which is the union of regular 
sheets. We denote by $M_{+}$the part of $M$ homeomorphic to $\Sigma$ via $p$, and by $M_{-}$the closure of the complementary part in $M$ :

$$
\begin{aligned}
& M_{+}=M \cap p^{-1}(\Sigma)=M \cap p^{-1}\left(\operatorname{cl}\left(\mathbb{R}^{n+1}-N(\Delta)\right)\right), \\
& M_{-}=M \cap p^{-1}(N(\Delta)) .
\end{aligned}
$$

As is usual with knot diagrams, we assume that $M_{+}$is contained in $\mathbb{R}^{n+1} \times$ $[0,1) \subset \mathbb{R}^{n+1} \times \mathbb{R}=\mathbb{R}^{n+2}$ and $M_{-}$is contained in $\mathbb{R}^{n+1} \times(-1,0]$. More precisely, we assume that

$$
\begin{gathered}
M_{+}=(\partial \Sigma) \times[0,1 / 2] \cup \Sigma \times\{1 / 2\}, \\
M_{-} \cap \mathbb{R}^{n+1} \times\{0\}=(\partial \Sigma) \times\{0\} .
\end{gathered}
$$

Take a base point $*$ in $\mathbb{R}^{n+2}=\mathbb{R}^{n+1} \times \mathbb{R}$ whose last coordinate is sufficiently large. For each $i \in\{1, \ldots, s\}$, consider a small arrow $a_{i}$ in $\mathbb{R}^{n+1}$ intersecting the interior of $\Sigma_{i}$ transversely in the direction of the co-orientation of $\Sigma_{i}$. Put a copy of $a_{i}$ in $\mathbb{R}^{n+1} \times\{0\}$, which we denote by the same symbol $a_{i}$. Let $x_{i}$ be an element of $\pi_{1}\left(\mathbb{R}^{n+1} \times[0, \infty)-M_{+}, *\right)$ represented by a loop $b_{i}^{0} \cdot a_{i} \cdot b_{i}^{1}$ in $\mathbb{R}^{n+1} \times[0, \infty)-M_{+}$with base point $*$, where $b_{i}^{0}$ is a straight path from $*$ to the initial point of $a_{i}$ and $b_{i}^{1}$ is a straight path from the terminal point of $a_{i}$ to $*$.

Lemma 4. (1) The element $x_{i} \in \pi_{1}\left(\mathbb{R}^{n+1} \times[0, \infty)-M_{+}, *\right)$ does not depend on the arrow $a_{i}$.

(2) The group $\pi_{1}\left(\mathbb{R}^{n+1} \times[0, \infty)-M_{+}, *\right)$ is a free group generated by $x_{1}, \ldots, x_{s}$.

Proof. (1) Let $a_{i}^{\prime}$ be another small arrow in $\mathbb{R}^{n+1}$ intersecting the interior of $\Sigma_{i}$ in the direction of the co-orientation of $\Sigma_{i}$, and let $x_{i}^{\prime}$ be an element of $\pi_{1}\left(\mathbb{R}^{n+1} \times[0, \infty)-M_{+}, *\right)$ obtained by use of $a_{i}^{\prime}$. Since $\Sigma_{i}$ is connected, we can slide the arrow $a_{i}^{\prime}$ onto $a_{i}$ along $\Sigma_{i}$; then $x_{i}^{\prime}$ is a conjugate of $x_{i}$ by an element represented by a loop in $\mathbb{R}^{n+1} \times[0, \infty)-M_{+}$which is disjoint from $\Sigma \times[0,1]$. Since the loop is null-homotopic in $\mathbb{R}^{n+1} \times[0, \infty)-M_{+}$, we have $x_{i}=x_{i}^{\prime}$.

(2) From the above argument we see that if $s=1$, then $\pi_{1}\left(\mathbb{R}^{n+1} \times\right.$ $\left.[0, \infty)-M_{+}, *\right)$ is a free group generated by $x_{1}$. We prove the assertion by induction on $s$. If $s>1$, consider a regular neighborhood $N\left(\Sigma_{1}\right)$ of $\Sigma_{1}$ in $\mathbb{R}^{n+1}$ and consider a cone $V_{1}$ in $\mathbb{R}^{n+1} \times[0, \infty)$ over the copy $\left(\partial N\left(\Sigma_{1}\right)\right) \times$ $\{0\}$ of $\partial N(\Sigma)$ with the base point $*$ as the cone vertex. The cone $V_{1}$ divides $\mathbb{R}^{n+1} \times[0, \infty)-M_{+}$into two pieces, say $H_{1}$ and $H_{2}$, such that $H_{1} \cap H_{2}=V_{1}$ and $H_{1}$ contains the component $M_{1}^{(1)}$ of $M_{+}$corresponding to $\Sigma_{1}$. It is not difficult to see that $H_{1}$ is a deformation retract of $\mathbb{R}^{n+1} \times[0, \infty)-M_{+}^{(1)}$ and $H_{2}$ is a deformation retract of $\mathbb{R}^{n+1} \times[0, \infty)-$ $\left(M_{+}-M_{+}^{(1)}\right)$. In particular, we have $\pi_{1}\left(H_{1}, *\right) \cong \pi_{1}\left(\mathbb{R}^{n+1} \times[0, \infty)-M_{+}^{(1)}, *\right)$ 
and $\pi_{1}\left(H_{2}, *\right) \cong \pi_{1}\left(\mathbb{R}^{n+1} \times[0, \infty)-\left(M_{+}-M_{+}^{(1)}\right), *\right)$. By induction hypothesis, the former is a free group generated by $x_{1}$ and the latter is a free group generated by $x_{2}, \ldots, x_{s}$. Applying the van Kampen theorem along $V_{1}$, we see that $\pi_{1}\left(\mathbb{R}^{n+1} \times[0, \infty)-M_{+}, *\right)$ is a free group generated by $x_{1}, \ldots, x_{s}$.

The images of $x_{1}, \ldots, x_{s}$ under the inclusion-induced homomorphism $\pi_{1}\left(\mathbb{R}^{n+1} \times[0, \infty)-M_{+}, *\right) \rightarrow \pi_{1}\left(\mathbb{R}^{n+2}-M, *\right)$ are denoted by the same symbols. They are the generators of the group presentation given in Theorem 2.

Proof of Theorem 2. Put $\Delta=\Delta^{1} \cup \Delta^{2}$, where $\Delta^{1}$ is the double point strata (the $(n-1)$-dimensional strata) and $\Delta^{2}$ is the lower dimensional strata. We divide the regular neighborhood $N(\Delta)$ as follows: Let $N\left(\Delta^{2}\right)$ be a regular neighborhood of $\Delta^{2}$ in $\mathbb{R}^{n+1}$, and put $W_{2}=\operatorname{cl}\left(\mathbb{R}^{n+1}-N\left(\Delta^{2}\right)\right)$. Let $N\left(\Delta^{1}\right)$ be a regular neighborhood of $\Delta^{1} \cap W_{2}$ in $W_{2}$, and put $W_{1}=$ $\operatorname{cl}\left(\mathbb{R}^{n+1}-N\left(\Delta^{2}\right)-N\left(\Delta^{1}\right)\right)$. We assume that $N(\Delta)$ is the union of $N\left(\Delta^{2}\right)$ and $N\left(\Delta^{1}\right)$.

Let $A_{1}$ and $A_{2}$ be arcs in a cylinder $D^{2} \times[-1,0]$ as in Figure 2. Notice that $N\left(\Delta^{1}\right)$ is a trivial $D^{2}$-bundle over $\Delta^{1} \cap W_{2}$, since each component of $\Delta^{1}$ (a double point stratum) has a trivialization determined from the four regular sheets around it. We identify $N\left(\Delta^{1}\right)$ with $\left(\Delta^{1} \cap W_{2}\right) \times D^{2}$ and $N\left(\Delta^{1}\right) \times[-1,0]$ with $\left(\Delta^{1} \cap W_{2}\right) \times\left(D^{2} \times[-1,0]\right)$. We may assume that $M_{-}$restricted to $N\left(\Delta^{1}\right) \times[-1,0]$ is $\left(\Delta^{1} \cap W_{2}\right) \times\left(A_{1} \cup A_{2}\right) \subset\left(\Delta^{1} \cap\right.$ $\left.W_{2}\right) \times\left(D^{2} \times[-1,0]\right)$. By a routine argument in knot theory using the van Kampen theorem inductively, we see that $\pi_{1}\left(\left(\mathbb{R}^{n+1} \times[0, \infty)-M_{+}\right)\right.$ $\left.\cup\left(N\left(\Delta^{1}\right) \times[-1,0]-M_{-}\right), *\right)$ has a group presentation as in Theorem 2 .

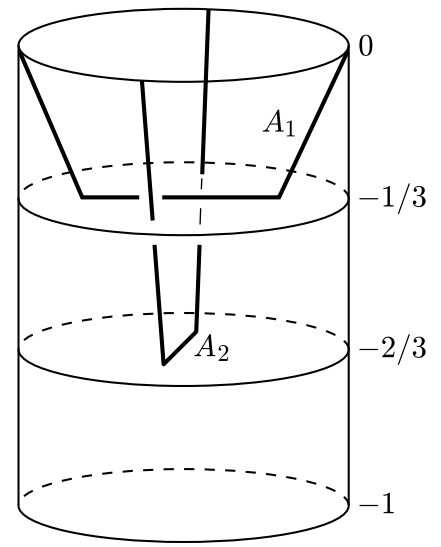

Fig. 2 
Since $\Delta^{2}$ has codimension more than two in $\mathbb{R}^{n+1}$, we have

$$
\begin{aligned}
& \pi_{1}\left(\mathbb{R}^{n+2}-M\right) \\
& \cong \pi_{1}\left(\left(\mathbb{R}^{n+1} \times[0, \infty)-M_{+}\right) \cup\left(\mathbb{R}^{n+1} \times(-\infty, 0]-M_{-}\right)-\Delta^{2} \times(-\infty, 0], *\right) \\
& \cong \pi_{1}\left(\left(\mathbb{R}^{n+1} \times[0, \infty)-M_{+}\right) \cup\left(\mathbb{R}^{n+1} \times[-1,0]-M_{-}\right)-N\left(\Delta^{2}\right) \times[-1,0], *\right) \\
& \cong \pi_{1}\left(\left(\mathbb{R}^{n+1} \times[0, \infty)-M_{+}\right) \cup\left(N\left(\Delta^{1}\right) \times[-1,0]-M_{-}\right), *\right) .
\end{aligned}
$$

Therefore the knot group $\pi_{1}\left(\mathbb{R}^{n+2}-M\right)$ has the required presentation.

\section{References}

[1] A. M. Brunner, E. J. Mayland Jr., and J. Simon, Knot groups in $S^{4}$ with nontrivial homology, Pacific J. Math. 103 (1982), 315-324.

[2] G. Burde and H. Zieschang, Knots, de Gruyter Stud. Math. 5, de Gruyter, 1985.

[3] J. S. Carter and M. Saito, Knotted Surfaces and their Diagrams, Math. Surveys Monographs 55, Amer. Math. Soc., 1998.

[4] R. H. Crowell and R. H. Fox, Introduction to Knot Theory, Grad. Texts in Math. 57, Springer, New York, 1977.

[5] R. H. Fox, A quick trip through knot theory, in: Topology of 3-Manifolds and Related Topics (Athens, GA, 1961), Prentice-Hall, 1962, 120-167.

[6] F. González-Acuña, A characterization of 2-knot groups, Rev. Mat. Iberoamericana 10 (1994), 221-228.

[7] C. McA. Gordon, Homology of groups of surfaces in the 4-sphere, Math. Proc. Cambridge Philos. Soc. 89 (1981), 113-117.

[8] J. A. Hillman, 2-Knots and Their Groups, Austral. Math. Soc. Lect. Ser. 5, Cambridge Univ. Press, 1989.

[9] S. Kamada, Non-orientable surfaces in 4-space, Osaka J. Math. 26 (1989), 367385.

[10] - A characterization of groups of closed orientable surfaces in 4-space, Topology 33 (1994), 113-122.

[11] A. Kawauchi, A Survey of Knot Theory, Birkhäuser, Basel, 1996.

[12] A. Kawauchi, T. Shibuya and S. Suzuki, Descriptions on surfaces in four-space, I. Normal forms, Math. Sem. Notes Kobe Univ. 10 (1982), 75-125.

[13] - - - - Descriptions on surfaces in four-space, II. Singularities and cross-sectional links, ibid. 11 (1983), 31-69.

[14] M. A. Kervaire, Les nœuds de dimensions supérieures, Bull. Soc. Math. France 93 (1965), 225-271.

[15] -, On higher dimensional knots, in: Differential and Combinatorial Topology, Princeton Math. Ser. 27, Princeton Univ. Press, 1965, 105-119.

[16] S. Kinoshita, Alexander polynomials as isotopy invariants I, Osaka Math. J. 10 (1958) 263-271.

[17] - On the Alexander polynomials of 2-spheres in a 4-sphere, Ann. of Math. 74 (1961), 518-531.

[18] R. A. Litherland, The second homology of the group of a knotted surface, Quart. J. Math. Oxford 32 (1981), 425-434.

[19] T. Maeda, On the groups with Wirtinger presentations, Math. Sem. Notes Kobe Univ. 5 (1977), 345-358.

[20] K. Reidemeister, Knotentheorie, Ergeb. Math. Grenzgeb. 1, Springer, 1932. 
[21] D. Rolfsen, Knots and Links, Publish or Perish, 1976.

[22] C. P. Rourke and B. J. Sanderson, Introduction to Piecewise-Linear Topology, Ergeb. Math. Grenzgeb. 69, Springer, 1972.

[23] J. Simon, Wirtinger approximations and the knot groups of $F^{n}$ in $S^{n+2}$, Pacific J. Math. 90 (1980), 177-190.

[24] S. Suzuki, Knotting problems of 2-spheres in the 4-sphere, Math. Sem. Notes Kobe Univ. 4 (1976), 241-371.

[25] T. Yajima, On the fundamental groups of knotted 2-manifolds in the 4-space, J. Math. Osaka City Univ. 13 (1962), 63-71.

[26] - On a characterization of knot groups of some spheres in $\mathbb{R}^{4}$, Osaka J. Math. 6 (1969), 435-446.

[27] -, Wirtinger presentations of knot groups, Proc. Japan Acad. 46 (1970), 9971000 .

Department of Mathematics

Osaka City University

Sumiyoshi, Osaka 558-8585, Japan

E-mail: kamada@sci.osaka-cu.ac.jp
Current addres:

Department of Mathematics and Statistics

University of South Alabama

Mobile, AL 36688, U.S.A.

E-mail: skamada@mathstat.usouthal.edu 